\title{
Effectiveness and Safety of Tissue Expander for Adjuvant Helical Tomotherapy in Curatively Resected Retroperitoneal Sarcoma
}

\author{
JEONG IL YU ${ }^{1}$, DO HOON LIM ${ }^{1}$, HEE CHUL PARK ${ }^{1}$, SANG HOON JUNG ${ }^{1}$, SEONG JIN JEON ${ }^{1}$, \\ HEERIM NAM ${ }^{2}$, BOKYOUNG KIM ${ }^{3}$, SUNG-JOO KIM ${ }^{4}$ and JAE BERM PARK ${ }^{4}$ \\ Departments of ${ }^{1}$ Radiation Oncology, and ${ }^{4}$ Surgery, Samsung Medical Center, Seoul, Republic of Korea; \\ ${ }^{2}$ Department of Radiation Oncology, Kangbuk Samsung Hospital, \\ Sungkyunkwan University School of Medicine, Seoul, Republic of Korea; \\ ${ }^{3}$ Department of Radiation Oncology, Sheikh Khalifa Specialist Hospital, Ras Al Khaimah, United Arab Emirates
}

\begin{abstract}
Background/Aim: We investigated the effectiveness and safety of using a tissue expander (TE) for adjuvant helical tomotherapy (HT) for curatively resected retroperitoneal sarcoma (RPS). Materials and Methods: This study was conducted with 60 RPS patients who underwent curative resection with or without TE insertion followed by HT from June 2009 to December 2016. Among the patients, TE was inserted in 37 (61.7\%). The quality of TE insertion was evaluated. Median follow-up after surgery was 19.4 months (range=4.5-93.2 months). Results: A higher biologically-equivalent dose $(\alpha / \beta=10)$ was used with patients who had TE insertion (median, 72.0 gray [Gy] vs. $67.1 \mathrm{~Gy}, p=0.02)$. The quality of TE insertion was excellent in 18 patients $(48.6 \%)$, good in 10 (27.0\%), fair in seven (18.9\%), and poor in two (5.4\%). Toxicity was not significantly different according to TE insertion. Local recurrence-free survival (LRFS) was $78.2 \%$, and overall survival was $95.6 \%$ at 3 years. TE insertion was not a significant factor for LRFS $(91.1 \%$ in TE vs. $62.9 \%$ in non$T E$ group at 3 years, $p=0.62$ ). In the subgroup of patients with Rl or unknown tumor margin status, however, LRFS was marginally higher in the TE insertion group $(100.0 \%$ in TE vs. $62.9 \%$ non-TE group at 3 years, $p=0.05)$. Conclusion: $H T$ with TE insertion as adjuvant $R T$ for curatively resected RPS was feasible with acceptable toxicity. In the patients
\end{abstract}

Correspondence to: Do Hoon Lim, Department of Radiation Oncology, Samsung Medical Center, Sungkyunkwan University School of Medicine, 81 Irwon-ro, Gangnam-gu, Seoul 06351, Republic of Korea. Tel: +82 234102612, Fax: +82 234102619, e-mail: dh8.lim@samsung.com

Key Words: Retroperitoneal sarcoma, radiotherapy, tissue expander, recurrence, toxicity. with RI or unknown tumor margin status, LRFS was marginally higher in the TE insertion group after HT.

Retroperitoneal sarcoma (RPS) is a relatively uncommon malignancy that accounts for approximately $10-15 \%$ of all soft tissue sarcomas (1). Because RPS occurs close to important organs, such as main vessels, kidneys, bowels, and liver, complete resection can be difficult. Local recurrence (LR) originating from this characteristic is one of the main factors in treatment failure (2).

Despite the lack of evidence from randomized controlled trials and based on positive outcomes from retrospective and prospective studies, radiotherapy (RT) is frequently used for RPS as a neoadjuvant and adjuvant modality for minimizing LR (3-6). In RPS, however, delivering sufficient RT doses for extremity sarcomas is challenging because of the radiation vulnerability of surrounding organs, especially the small bowel (7). However, several strategies have been suggested to overcome the radiation weakness in the small bowel.

Strategies to overcome the radiation vulnerability of surrounding normal organs and deliver optimal RT doses for local control of RPS include preoperative, intraoperative, more precise RT including intensitymodulated RT (IMRT) or stereotactic body RT (SBRT), and particle beam RT (8-10). Our institution is attempting helical tomotherapy (HT) and tissue expander (TE) insertion methods. HT, a form of IMRT, is an optimized technique for radiation delivery in a helical pattern using a linear accelerator with a rotating gantry $(11,12)$. Using this high-precision technique, HT irradiates tumors selectively with relative sparing of surrounding normal organs. TE insertion serves to separate surrounding normal organs from the tumor bed that is the target area for high-dose RT in order to minimize LR $(11,12)$. 
We conducted this study to evaluate the efficacy and safety of HT with TE insertion as adjuvant RT for curatively resected RPS.

\section{Materials and Methods}

Patients. This study was approved by the Samsung Medical Center Institutional Review Board (SMC IRB 2017-07-026), and written informed consent was waived by the board. This retrospective study included patients in the Samsung Medical Center Radiation Oncology registry who were treated with adjuvant HT for curatively resected RPS from June 2009 to December 2016. Patients were included even if they had remaining lesions as long as most of their tumor was resected with curative intent.

Surgical resection and tissue expander insertion. TE insertion was recommended to patients who were likely to need adjuvant RT. TE insertion was considered more aggressively for patients for whom safe delivery of an optimal RT dose was considered to be difficult, including patients with multiple resection for recurrent and larger tumors. Before surgical resection, informed consent for surgical resection with TE insertion was obtained from all patients. A TE was inserted in patients who agreed to it because the device was not covered by the Korean national insurance program.

Detailed surgical methods of resection and TE insertion were described in a previous article (5). Briefly, en bloc curative surgical resection, including adjacent organs invaded by the tumor, was performed in all RPS patients. Major vascular resection was not performed for tumors invading major arteries, including the aorta, celiac axis, or superior mesenteric artery. If the patient had an indication for TE insertion, one or two TEs (Tissue Expander LSRT 83 and 82 series; SEBBIN, Boissy L'Aillerie, France) filled with saline were placed in the retroperitoneal tumor bed after resection. Two or three suturing procedures were conducted to prevent movement of the TE before or during adjuvant RT.

Adjuvant radiotherapy. Details of our institutional protocol for adjuvant RT for RPS sarcoma were introduced in a previous study (13). Adjuvant RT was generally started 5 to 7 weeks after surgical resection. CT scans with intravenous contrast agent for RT treatment planning were performed on all patients, generally in a supine position with the arm over the head.

Target delineation used the Pinnacle3 Treatment Planning System (Philips Medical Systems, Andover, MA). Gross tumor volume (GTV) was defined as gross residual lesion and/or high risk area of resection margin as indicated by the surgeon for R1/R2-resected patients. CTV was defined as RPS attached area on the retroperitoneal surface except for the bowel and was restricted to the dorsal half of the flank side of the abdominal cavity. Planning target volume (PTV) was determined by adding 5 to $10 \mathrm{~mm}$ to CTV.

After target delineation, final data were transferred to an HT planning work station for treatment planning with a TomoTherapy System (Accuray Inc., Madison, WI, USA). The RT dose goal was 60 to 66 Gy to the GTV and 50 to 54 Gy to the CTV as a single fraction of 2 to $2.5 \mathrm{~Gy}$. Final dose was restricted by limitations of normal tissue constraints, including bowel dose as $55 \mathrm{~Gy}$ of maximum dose and volume of $65 \mathrm{cc}$ exposed above $45 \mathrm{~Gy}$. Other dose constraints, including those of the spine, kidney, liver, and skin, were set according to the Quantitative Analyses of Normal Tissue Effects in the Clinic recommendation (14). For removed single kidneys abutting and/or invaded by RPS, a more stringent restriction was applied as less than $10 \mathrm{~Gy}$ to more than two-thirds of the remaining kidney.

Adjuvant chemotherapy. Adjuvant chemotherapy for complete resected RPS was not routinely recommended during the study period. Chemotherapy was selectively recommended to patients with differentiated liposarcoma with larger than $20 \mathrm{~cm}$ diameter or leiomyosarcoma. It was also considered for patients with sarcoma of French Fédération Nationale des Centres de Lutte Contre le Cancer (FNCLCC) grade 3.

Evaluation of clinical outcomes and follow-up. Quality evaluation of TE insertion was performed as described in a previous study using planning CT images registered in the Pinnacle treatment planning system (Philips Radiation Oncology Systems, Milpitas, CA, USA) based on correlation of retroperitoneal surface volume covered by TE with CTV.11 Quality of TE insertion was divided into excellent $(\geq 85 \%)$, good $(70-85 \%)$, fair $(50-70 \%)$, and poor $(<50 \%)$ according to the ratio of retroperitoneal surface to CTV and TE.

During RT, patients were routinely examined at least once a week. After RT completion, follow-up with abdominopelvic CT scans was performed every two to three months for the first two years, every six months for the next three years, then yearly. Assessment of treatment-related adverse events was according to the Common Terminology Criteria for Adverse Events version 4.0 (15).

LR was defined as tumor recurrence within CTV, which was the surface area of the retroperitoneal side of the TE. Other recurrence was classified as distant metastasis, including recurrences associated with the bowel wall. More than two sites of recurrence detected within four weeks was defined as simultaneous recurrence.

Statistical analysis. To compare characteristics between TE and non-TE groups, Chi-square, Fisher's exact, or Mann-Whitney test was used. LRFS, RFS, and OS were measured from date of surgical resection to date of event detection or date of last follow-up visit. The Kaplan-Meier method was used to estimate survival rates. To compare LRFS, RFS, and OS according to clinicopathological variables including TE insertion, the log-rank test was used. SPSS 23.0 software for Windows (IBM, Armonk, NY) was used for statistical analysis. All $p$-values were two-sided, and $p<0.05$ was considered statistically significant.

\section{Results}

Patients. During the study period, 110 RPS patients received RT in our Department. Among them, HT was performed on 61. Except for one patient who received palliative surgery, 60 patients were finally included, and TE was inserted in 37 $(61.7 \%)$.

Baseline clinicopathological characteristics of patients according to TE insertion are in Table I. Median patient age was not different between the groups: 55 years (range $=34$ 78 years) for the TE insertion group and 53 years (range $=33$ 68 years) for the non-TE insertion group. No significant differences were observed in sex, recurrent presentation, or FNCLCC grade between the two groups. Significant differences were observed in tumor size $(p=0.05)$, 
Table I. Baseline clinicopathological characteristic of 60 study patients.

\begin{tabular}{|c|c|c|c|}
\hline Variables & $\begin{array}{c}\mathrm{TE} \\
(\mathrm{n}=37)\end{array}$ & $\begin{array}{c}\text { Non-TE } \\
(\mathrm{n}=23)\end{array}$ & $p$-Value \\
\hline \multicolumn{4}{|l|}{ Age-year } \\
\hline Median & 55 & 53 & \multirow[t]{2}{*}{0.49} \\
\hline Range & $34-78$ & $33-68$ & \\
\hline \multicolumn{4}{|l|}{ Gender-n $(\%)$} \\
\hline Male & $19(51.4)$ & $9(39.1)$ & 0.26 \\
\hline \multicolumn{4}{|l|}{ Presentation } \\
\hline Primary & $26(70.3)$ & 19 (82.6) & \multirow[t]{2}{*}{0.37} \\
\hline Recurrent & $11(29.7)$ & $4(17.4)$ & \\
\hline \multicolumn{4}{|l|}{ Tumor size $(\mathrm{cm})$} \\
\hline Median & 17.0 & 10.5 & \multirow[t]{2}{*}{0.05} \\
\hline Range & $2.0-50.0$ & $2.0-34.0$ & \\
\hline \multicolumn{4}{|l|}{ Completeness of resection } \\
\hline R0 & $2(5.4)$ & $5(21.7)$ & \multirow[t]{4}{*}{0.05} \\
\hline $\mathrm{R} 1$ & $18(48.6)$ & $9(39.1)$ & \\
\hline $\mathrm{R} 2$ & $6(16.2)$ & $0(0.0)$ & \\
\hline Unknown & $11(29.7)$ & $9(39.1)$ & \\
\hline \multicolumn{4}{|l|}{ FNCLCC tumor grade } \\
\hline 1 & $15(40.5)$ & $8(34.8)$ & \multirow[t]{3}{*}{0.34} \\
\hline 2 & $18(48.6)$ & $9(39.1)$ & \\
\hline 3 & $4(10.8)$ & $6(26.1)$ & \\
\hline \multicolumn{4}{|l|}{ Histology } \\
\hline Well differentiated liposarcoma & $14(37.8)$ & $4(17.4)$ & \\
\hline Dedifferentiated liposarcoma & $20(54.1)$ & $12(52.2)$ & \\
\hline Leiomyosarcoma & $0(0.0)$ & $7(30.4)$ & \\
\hline Other & $3(8.1)$ & $0(0.0)$ & 0.001 \\
\hline \multicolumn{4}{|l|}{$\operatorname{BED}(\alpha / \beta=10)$} \\
\hline Median (Gy) & 72.0 & 67.1 & \multirow[t]{2}{*}{0.02} \\
\hline Range (Gy) & $60.0-80.5$ & $45.6-75.0$ & \\
\hline
\end{tabular}

TE: Tissue expander; FNCLCC: French Fédération Nationale des Centres de Lutte Contre le Cancer; BED: biologically equivalent dose.

completeness of resection $(p=0.05)$, and histological subtype $(p=0.001)$. In the TE group, compared to the non-TE group, tumor size was larger (median $17 \mathrm{~cm} \mathrm{vs.} 10.5 \mathrm{~cm}$ ), and R2 resection/well-differentiated liposarcoma histology was more common. R0 resection and leiomyosarcoma histology were more common in the non-TE group. Median total delivered RT dose was significantly higher, at 72 gray (Gy), for the TE group than the $67.1 \mathrm{~Gy}$ for the non-TE group measured in biologically equivalent dose (BED) using $\alpha / \beta 10(p=0.02)$.

Quality of TE insertion. The quality of TE insertion was excellent in 18 patients $(48.6 \%)$, good in $10(27.0 \%)$, fair in seven $(18.9 \%)$, and poor in two $(5.4 \%)$, when 37 patients with TE were classified according to our evaluation criteria. In two patients evaluated as receiving poor quality TE insertion, TE was deflated before RT.

Adverse events. No treatment-related deaths or severe toxicities of grade 4 or higher were observed. Acute and chronic adverse events caused by TE insertion are listed in
Table II. Acute adverse events were slightly more common in the TE group, and more than three days of RT interruption was also higher in the TE group (8.1\% in TE vs. $4.3 \%$ in non-TE group). Differences between groups were not significant. Most events were grade 1 or 2 and were mostly alleviated within several weeks of treatment end.

As a chronic adverse event, abdominal pain of grade 1 or 2 was not different between the two groups. Severe toxicities that required surgical treatment were slightly higher in the TE group (13.5\% in TE vs. 8.7\% in non-TE group), but there was no statistically significant difference.

Patterns of failure. During follow-up, 21 patients $(35.0 \%)$ experienced some type of recurrence with a median of 16.5 months (range=2.6-39.6 months) from operation to recurrence. LR was detected in eight patients with a median of 31.0 months (range=18.3-39.6 months) from operation to LR. Three of these eight patients with LR also showed peritoneal seeding, three others had distant organ metastasis (liver in two, liver and lung in one), and the remaining patients had RT outfield recurrence.

Recurrence developed in 11 patients $(29.7 \%)$ in the TE group and $10(43.5 \%)$ in the non-TE group, and LR was present in four patients in each group. Among them, LR was detected in three patients who had received more than $70 \mathrm{~Gy}$ of BED, and all of these patients were $\mathrm{R} 2$ resected cases. Isolated recurrence was seen from the bowel surface initially attached to the primary tumor and the medial side of the TE in two patients with TE insertion; these patients had received curative surgical resection, and the operator confirmed that the recurrent tumor had aggressively invaded the bowel. The recurrent tumor was resected along with the invaded bowel, and the patients were followed for four and eight months after salvage surgery without evidence of disease recurrence.

Survival and prognostic factors. For all enrolled patients at 3 years after surgery, OS was $95.6 \%$, RFS was $52.9 \%$ and LRFS was $78.2 \%$. Kaplan-Meier curves for OS, RFS, and LRFS are shown in Figure 1. For the TE group, 3-year OS was $95.2 \%$, RFS was $65.7 \%$, and LRFS was $91.1 \%$; for the non-TE group, OS was $95.7 \%$, RFS was $34.7 \%$, and LRFS was $62.9 \%$. No significant differences between groups were seen for any survival curves.

Outcomes for probable prognostic factor analysis of LRFS and RFS are listed in Table III. LRFS was significantly affected by completeness of resection at 3 years, at $100.0 \%$ for patients receiving $\mathrm{R} 0$ resection, $77.4 \%$ for $\mathrm{R} 1$ resection or unknown margin status, and $62.5 \%$ for $\mathrm{R} 2$ resection $(p=0.02)$. FNCLCC grade was the most significant prognostic factor for RFS at 3 years, at $76.3 \%$ for patients with grade $1,54.5 \%$ for grade 2 , and $0.0 \%$ for grade 3 $(p<0.001)$. Kaplan-Meier curves for LRFS and RFS according to these factors are presented in Figure 2. 
Table II. Acute and chronic adverse events.

\begin{tabular}{|c|c|c|c|c|c|}
\hline \multirow[t]{2}{*}{ Events } & \multicolumn{2}{|c|}{ Gr I or II } & \multicolumn{2}{|c|}{ Grade III } & \multirow[t]{2}{*}{$p$-Value } \\
\hline & $\mathrm{TE}(\mathrm{n}=37)$ & Non-TE $(n=23)$ & TE $(n=37)$ & Non-TE $(n=23)$ & \\
\hline Fatigue & $5(13.5)$ & $1(4.3)$ & - & - & 0.39 \\
\hline Anorexia & $19(51.4)$ & $11(47.8)$ & $1(2.7)$ & - & 0.87 \\
\hline Nausea & $26(70.3)$ & $12(52.2)$ & - & - & 0.18 \\
\hline Vomiting & $11(29.7)$ & $3(13.0)$ & - & - & 0.21 \\
\hline Diarrhea & $9(24.3)$ & $6(26.1)$ & $1(2.7)$ & - & 1.00 \\
\hline RT interruption & $3(8.1)$ & $1(4.3)$ & - & - & 1.00 \\
\hline Abdominal pain & $12(32.4)$ & $11(47.8)$ & $1(2.7)$ & - & 0.40 \\
\hline Bowel toxicities & - & - & $5(13.5)$ & $2(8.7)$ & 0.70 \\
\hline
\end{tabular}

TE: Tissue expander; RT: radiotherapy.

TE insertion itself was not a significant prognostic factor for LRFS or RFS. In the subgroup analysis with the patients with $\mathrm{R} 1$ resection or unknown margin status, however, there was a difference in clinical outcomes according to TE insertion. The total dose of RT was significantly higher in the TE group (median BED $72 \mathrm{~Gy}$, range=60-81 Gy) than in the non-TE group (median BED $69 \mathrm{~Gy}$, range=46-72 Gy) $(p=0.04)$. In addition, LRFS at 3 years was higher in the TE group at $100.0 \%$ versus $62.9 \%$ in the non-TE group $(p=0.05)$. Kaplan-Meier curves for LRFS according to TE insertion for patients with $\mathrm{R} 1$ resection or unknown margin status are presented in Figure 3.

\section{Discussion}

This study evaluated the efficacy and safety of HT with TE insertion as adjuvant RT for curatively resected RPS. A favorable LRFS of $78.2 \%$ at 3 years and acceptable toxicities were observed. About $95 \%$ of patients who underwent TE insertion had more than $50 \%$ clinical target volume (CTV) coverage by TE, and a significantly higher radiation dose was delivered in the TE group. Although TE insertion was not associated with higher LRFS in all patients, the subgroup of patients with $\mathrm{R} 1$ resection or unknown margin status tended to have higher RT dose and LRFS after TE insertion. RPS is a rare malignancy with an incidence of about 3.4 cases per 1 million person-years and accounts for about $6.6 \%$ of soft tissue sarcomas (16). Surgical resection is standard management, but complete resection is not easy because of anatomical locations, and LR is common (17). Although surgical management has led to major advances in efforts to standardize and improve surgical quality of RPS, approximately $30 \%$ LR was still reported in a large cohort study (18).

The necessity of RT is still controversial for RPS management. However, obtaining maximum local control is important, and the necessity of appropriate RT as an adjunctive therapy is widely recognized (19-21). Nonetheless, adjuvant RT for RPS has limitations such as the low radiosensitivity of sarcomas and their proximity to organs that are vulnerable to radiation. In addition, tumor sizes are often larger than $20 \mathrm{~cm}$, making difficult uniform and precise highdose RT. In addition, the area of risk included in the RT target is expanded after surgical resection. Recent guidelines recommend neoadjuvant $\mathrm{RT}$ rather than adjuvant RT for RPS because of these problems $(22,23)$. However, at the time of neoadjuvant RT, obtaining accurate pathologic results is difficult, and concerns about excessive treatment exist.

TE can reduce bowel exposure, which is the major obstacle to delivering high-dose RT. TE prevents natural repositioning of the bowel by replacing the area of previous RPS (24, 25). Our group previously reported on the effectiveness and safety of TE insertion for adjuvant RT of RPS (13). In this subsequent study, the quality of TE insertion was higher than in the previous study because of the learning curve related to TE insertion. We reaffirmed that a significantly higher radiation dose could be delivered without significant differences in acute and late toxicities for a TE group compared to a non-TE group, even for larger tumors in the TE group. However, the two recurrent cases that might have been irradiated with high doses of RT and could be classified as local recurrence if we did not use TE developed because very precise irradiation was administered to the retroperitoneal surface. To prevent this kind of recurrence, initial aggressive resection might be needed. Physicians need to consider the possibility of such recurrence after TE insertion and high-precision RT.

$\mathrm{HT}$ is a technique for delivering slice-by-slice radiation using a linear accelerator attached to a rotating gantry. HT lacks the limitations of conventional RT techniques such as RT range and dose inhomogeneity of a large RT field (11). Even though RT has a wide range, HT allows control of the 


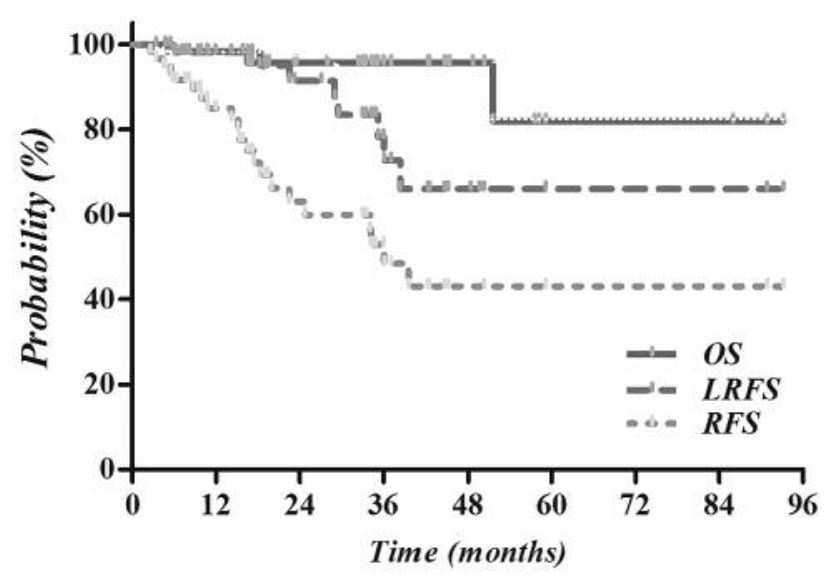

Figure 1. Kaplan-Meier curves for OS, RFS, and LRFS of all patients.
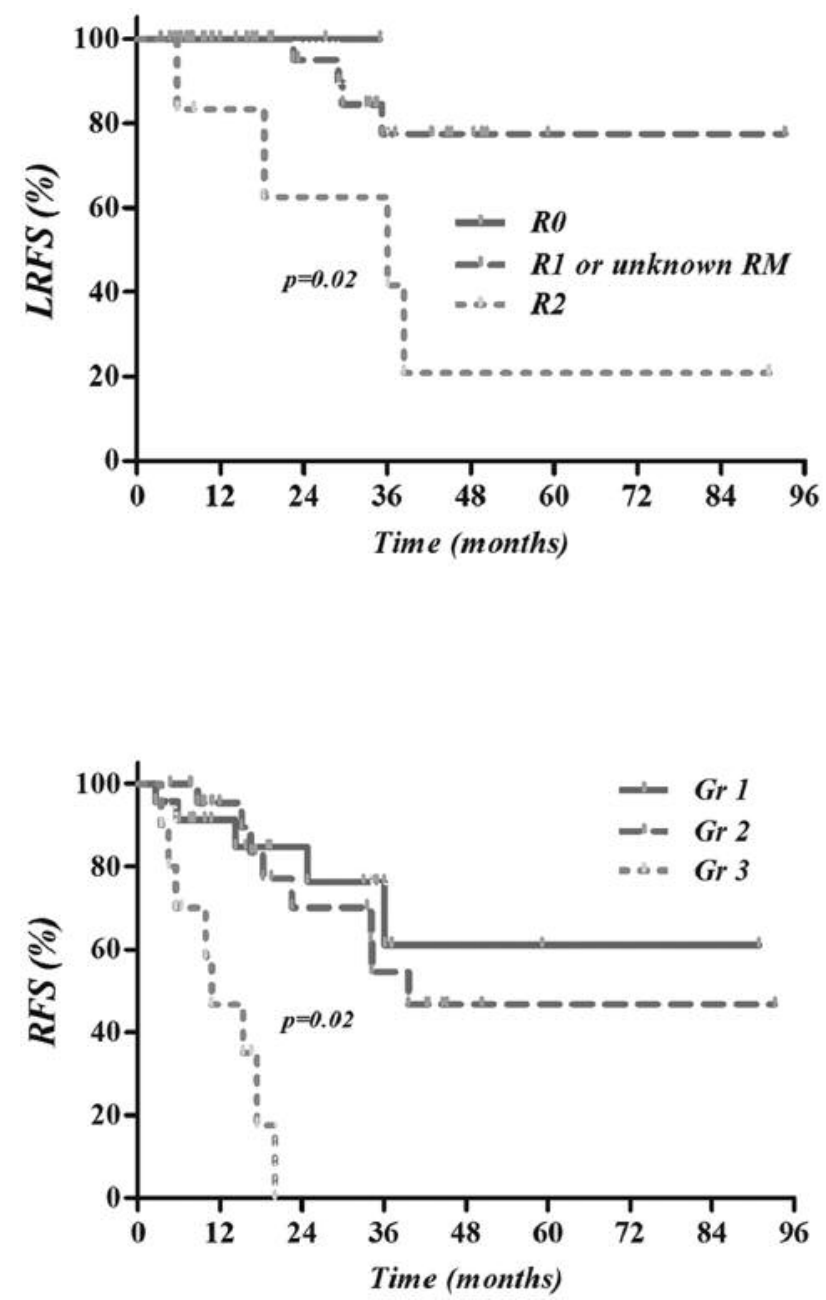

Figure 2. Kaplan-Meier curves for LRFS and RFS according to prognostic factors. LRFS was significantly affected by completeness of resection (A), and RFS was significantly affected by FNCLCC grade (B).

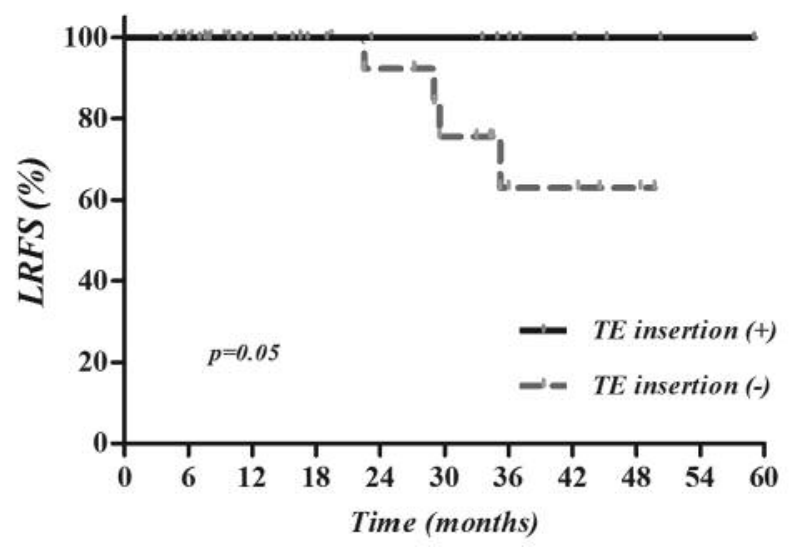

Figure 3. Kaplan-Meier curves for LRFS for patients with R1 or unknown margin status. TE insertion was associated with marginal difference in LRFS.

Table III. Possible prognostic factors of local recurrence-free survival for curatively resected retroperitoneal sarcoma.

\begin{tabular}{|c|c|c|c|c|c|}
\hline Variables & No & 3-y LRFS (\%) & $p$-Value & 3-yr RFS & $p$-Value \\
\hline \multicolumn{6}{|l|}{ Gender } \\
\hline Male & 28 & 90.0 & \multirow[t]{2}{*}{0.30} & 54.6 & \multirow[t]{2}{*}{0.77} \\
\hline Female & 32 & 69.3 & & 49.8 & \\
\hline \multicolumn{6}{|l|}{ Age (yrs) } \\
\hline$\leq 55$ & 34 & 81.6 & \multirow[t]{2}{*}{0.79} & 51.6 & \multirow[t]{2}{*}{0.63} \\
\hline$>55$ & 26 & 69.7 & & 55.6 & \\
\hline \multicolumn{6}{|l|}{ Presentation } \\
\hline Primary & 45 & 78.7 & \multirow[t]{2}{*}{0.33} & 54.0 & \multirow[t]{2}{*}{0.14} \\
\hline Recurrent & 15 & 76.9 & & 45.4 & \\
\hline \multicolumn{6}{|l|}{ Pathology } \\
\hline Liposarcoma & 50 & 75.5 & \multirow[t]{2}{*}{0.26} & 56.0 & \multirow[t]{2}{*}{0.37} \\
\hline Others & 10 & 100.0 & & 30.9 & \\
\hline \multicolumn{6}{|l|}{ Tumor size } \\
\hline$<15 \mathrm{~cm}$ & 27 & 61.5 & \multirow[t]{2}{*}{0.51} & 31.0 & \multirow[t]{2}{*}{0.01} \\
\hline$\geq 15 \mathrm{~cm}$ & 33 & 84.7 & & 65.7 & \\
\hline \multicolumn{6}{|l|}{ FNCLCC grade } \\
\hline 1 & 23 & 95.7 & \multirow[t]{3}{*}{0.80} & 76.3 & \multirow[t]{3}{*}{$<0.001$} \\
\hline 2 & 27 & 70.2 & & 54.5 & \\
\hline 3 & 10 & 50.0 & & 0.0 & \\
\hline \multicolumn{6}{|l|}{$\begin{array}{l}\text { Completeness } \\
\text { of resection }\end{array}$} \\
\hline R0 & 6 & 100.0 & \multirow[t]{3}{*}{0.02} & 57.1 & \multirow[t]{3}{*}{0.76} \\
\hline $\mathrm{R} 1 / \mathrm{UK}$ & 48 & 77.4 & & 49.5 & \\
\hline $\mathrm{R} 2$ & 6 & 62.5 & & 62.5 & \\
\hline \multicolumn{6}{|l|}{ TE insertion } \\
\hline No & 23 & 62.9 & \multirow[t]{2}{*}{0.62} & 34.7 & \multirow[t]{2}{*}{0.42} \\
\hline Yes & 37 & 91.1 & & 65.7 & \\
\hline \multicolumn{6}{|l|}{$\operatorname{BED}(\alpha / \beta=10)$} \\
\hline$<72$ Gy & 32 & 74.8 & \multirow[t]{2}{*}{0.79} & 54.2 & \multirow[t]{2}{*}{0.39} \\
\hline$\geq 72 \mathrm{~Gy}$ & 28 & 100.0 & & 61.2 & \\
\hline
\end{tabular}

LRFS: Local recurrence-free survival; RFS: recurrence-free survival; FNCLCC: French Fédération Nationale des Centres de Lutte Contre le Cancer; UK: unknown resection margin status; TE: tissue expander; BED: biologically-equivalent dose. 
dose of each slice in order to deliver precise radiation doses. Because of these advantages, our institution routinely performs HT for RPS for precise delivery of radiation to a CTV distributed over a large area and involving body flexion. Unlike previous studies, this study found that the ability to deliver higher RT dose to both a TE and a non-TE group might be associated with HT advantages. A dosimetric study of RPS with large CTV confirmed the superiority of HT for dose uniformity compared to step-and-shoot IMRT $(9,26)$. In addition, HT is optimized for image guidance using megavoltage CT (MVCT) images in an IMRT era where importance of image guidance is more emphasized (27).

Despite the theoretical and dosimetric advantages of HT for RPS, clinical studies using HT for RPS are rarely reported. In one study, HT as preoperative RT for RPS was feasible for delivering high-dose radiation with acceptable toxicities (11). Also, in that study, HT showed superior dosimetric outcomes compared to IMRT. In addition, several studies have reported clinical outcomes for IMRT for RPS, but most were in a neoadjuvant rather than an adjuvant setting $(4,10,28)$.

This study had several limitations. First, it was a retrospective analysis from a single institution and so cannot be free from selection bias. TE insertion was not covered by Korean national insurance, so selection of TE insertion could also be biased. TE insertion quality must be interpreted carefully because its learning curve primarily occurred before the study period of 2012 to 2016 .

To the best of our knowledge, this study is the first to report the efficacy and side-effects of HT after TE insertion for curatively resected RPS patients. Although the trend toward RPS management focuses on neoadjuvant RT, the effect of adjuvant RT has been repeatedly confirmed. If biopsy results are repeatedly uncertain or the need for RT is confirmed during surgery, adjuvant RT should be considered. In addition, this RT might be an attractive option for HT after TE insertion in such situations. Further prospective studies are needed to confirm the results of our study.

In conclusion, HT with TE insertion for adjuvant RT for curatively resected RPS showed feasible outcomes with acceptable toxicities. In most patients, TE insertion was helpful for delivering higher RT dose covering more than $50 \%$ of the CTV. TE insertion was related to significantly higher RT dose delivery and marginally significantly higher LRFS in the subgroup of patients with $\mathrm{R} 1$ resection or unknown margin status.

\section{Conflicts of Interest}

The Authors declare that they have no conflicts of interest related to this work.

\section{Acknowledgements}

This research was supported by a Basic Science Research Program through the National Research Foundation of Korea (NRF), which was funded by the Ministry of Education (NRF-2017R1D1 A1B03031275), and a grant from the Marine Biotechnology Program (20150220), which was funded by the Ministry of Oceans and Fisheries, Korea.

\section{References}

1 Jemal A, Siegel R, Ward E, Murray T, Xu J and Thun MJ: Cancer statistics, 2007. CA Cancer J Clin 57: 43-66, 2007.

2 Strauss DC, Hayes AJ, Thway K, Moskovic EC, Fisher C and Thomas JM: Surgical management of primary retroperitoneal sarcoma. Br J Surg 97: 698-706, 2010.

3 Lee HS, Yu JI, Lim DH and Kim SJ: Retroperitoneal liposarcoma: the role of adjuvant radiation therapy and the prognostic factors. Radiat Oncol J 34: 216-222, 2016.

4 Ecker BL, Peters MG, McMillan MT, Sinnamon AJ, Zhang PJ, Fraker DL, Levin WP, Roses RE and Karakousis GC: Preoperative radiotherapy in the management of retroperitoneal liposarcoma. Br J Surg 103: 1839-1846, 2016.

5 Cassier PA, Kantor G, Bonvalot S, Lavergne E, Stoeckle E, Le Pechoux C, Meeus P, Sunyach MP, Vaz G, Coindre JM, Linassier C, Labib A, Delcambre C, Bay JO, Leyvraz S, Duberge T, Lagrange JL, Duret A and Blay JY: Adjuvant radiotherapy for extremity and trunk wall atypical lipomatous tumor/well-differentiated LPS (ALT/WD-LPS): a French Sarcoma Group (GSF-GETO) study. Ann Oncol 25: 1854-1860, 2014.

6 Choi AH, Barnholtz-Sloan JS and Kim JA: Effect of radiation therapy on survival in surgically resected retroperitoneal sarcoma: a propensity score-adjusted SEER analysis. Ann Oncol 23: 2449-2457, 2012.

7 Bae SH, Kim MS, Cho CK, Kang JK, Lee SY, Lee KN, Lee DH, Han CJ, Yang KY and Kim SB: Predictor of severe gastroduodenal toxicity after stereotactic body radiotherapy for abdominopelvic malignancies. Int J Radiat Oncol Biol Phys 84 : e469-474, 2012.

8 Wang LB, McAneny D, Doherty G and Sachs T: Effect of intraoperative radiotherapy in the treatment of retroperitoneal sarcoma. Int J Clin Oncol 22: 563-568, 2017.

9 Ostheimer C, Hubsch P, Janich M, Gerlach R and Vordermark D: Dosimetric comparison of intensity-modulated radiotherapy (IMRT) and volumetric modulated arc therapy (VMAT) in total scalp irradiation: a single institutional experience. Radiat Oncol J 34: 313-321, 2016.

10 Roeder F, Ulrich A, Habl G, Uhl M, Saleh-Ebrahimi L, Huber PE, Schulz-Ertner D, Nikoghosyan AV, Alldinger I, Krempien R, Mechtersheimer G, Hensley FW, Debus J and Bischof M: Clinical phase I/II trial to investigate preoperative doseescalated intensity-modulated radiation therapy (IMRT) and intraoperative radiation therapy (IORT) in patients with retroperitoneal soft tissue sarcoma: interim analysis. BMC Cancer 14: 617, 2014.

11 Sargos P, Dejean C, de Figueiredo BH, Brouste V, Nguyen Bui B, Italiano A, Stoeckle E and Kantor G: High-dose pre-operative helical tomotherapy (54 Gy) for retroperitoneal liposarcoma. Radiat Oncol 7: 214, 2012. 
12 Pezner RD, Liu A, Han C, Chen YJ, Schultheiss TE and Wong JY: Dosimetric comparison of helical tomotherapy treatment and step-and-shoot intensity-modulated radiotherapy of retroperitoneal sarcoma. Radiother Oncol 81: 81-87, 2006.

13 Yu JI, Lim DH, Park HC, Nam H, Kim BK, Kim SJ and Park JB: Clinical outcomes of tissue expanders on adjuvant radiotherapy of resected retroperitoneal sarcoma. Medicine (Baltimore) 95: e4123, 2016.

14 Marks LB, Yorke ED, Jackson A, Ten Haken RK, Constine LS, Eisbruch A, Bentzen SM, Nam J and Deasy JO: Use of normal tissue complication probability models in the clinic. Int J Radiat Oncol Biol Phys 76: S10-19, 2010.

15 National Cancer Institute: Common Terminology Criteria for Adverse Events (CTCAE) Version 4.0. Bethesda, MD, U.S Department of Health and Human Services, 2009.

16 Jemal A, Siegel R, Ward E, Hao Y, Xu J and Thun MJ: Cancer statistics, 2009. CA Cancer J Clin 59: 225-249, 2009.

17 Toulmonde M, Bonvalot S, Meeus P, Stoeckle E, Riou O, Isambert N, Bompas E, Jafari M, Delcambre-Lair C, Saada E, Le Cesne A, Le Pechoux C, Blay JY, Piperno-Neumann S, Chevreau C, Bay JO, Brouste V, Terrier P, Ranchere-Vince D, Neuville A and Italiano A: Retroperitoneal sarcomas: patterns of care at diagnosis, prognostic factors and focus on main histological subtypes: a multicenter analysis of the French Sarcoma Group. Ann Oncol 25: 735-742, 2014.

18 Smith HG, Panchalingam D, Hannay JA, Smith MJ, Thomas JM, Hayes AJ and Strauss DC: Outcome following resection of retroperitoneal sarcoma. Br J Surg 102: 1698-1709, 2015.

19 Hull MA, Molina G, Niemierko A, Haynes AB, Jacobson A, Bernstein KA, Chen YL, DeLaney TF and Mullen JT: Improved local control with an aggressive strategy of preoperative (with or without intraoperative) radiation therapy combined with radical surgical resection for retroperitoneal sarcoma. J Surg Oncol 115: 746-751, 2017.

20 Kelly KJ, Yoon SS, Kuk D, Qin LX, Dukleska K, Chang KK, Chen YL, Delaney TF, Brennan MF and Singer S: Comparison of perioperative radiation therapy and surgery versus surgery alone in 204 patients with primary retroperitoneal sarcoma: a retrospective 2-institution study. Ann Surg 262: 156-162, 2015.

21 Nussbaum DP, Rushing CN, Lane WO, Cardona DM, Kirsch DG, Peterson BL and Blazer DGR: Preoperative or postoperative radiotherapy versus surgery alone for retroperitoneal sarcoma: a case-control, propensity score-matched analysis of a nationwide clinical oncology database. Lancet Oncol 17: 966-975, 2016.
22 Management of Recurrent Retroperitoneal Sarcoma (RPS) in the Adult: A Consensus Approach from the Trans-Atlantic RPS Working Group. Ann Surg Oncol 23: 3531-3540, 2016.

23 National Comprehensive Cancer Network. NCCN Clinical Practice Guidelines in Oncology 2017. https://www.nccn.org/ professionals/physician_gls/default.aspx.

24 White JS, Biberdorf D, DiFrancesco LM, Kurien E and Temple W: Use of tissue expanders and pre-operative external beam radiotherapy in the treatment of retroperitoneal sarcoma. Ann Surg Oncol 14: 583-590, 2007.

25 Park H, Lee S, Kim B, Lim do H, Choi YL, Choi GS, Kim JM, Park JB, Kwon CH, Joh JW and Kim SJ: Tissue expander placement and adjuvant radiotherapy after surgical resection of retroperitoneal liposarcoma offers improved local control. Medicine (Baltimore) 95: e4435, 2016.

26 Taggar AS, Graham D, Kurien E and Grafe JL: Volumetricmodulated arc therapy versus intensity-modulated radiotherapy for large volume retroperitoneal sarcomas: A comparative analysis of dosimetric and treatment delivery parameters. J Appl Clin Med Phys 19: 276-281, 2018.

27 Sasaki N, Yamazaki H, Shimizu D, Suzuki D, Masui K, Nakamura S, Okabe H, Nihikawa T and Yoshida K: Long-term outcomes of a dose-reduction trial to decrease late gastrointestinal toxicity in patients with prostate cancer receiving soft tissue-matched image-guided intensity-modulated radiotherapy. Anticancer Res 38: 385-391, 2018.

28 Yoon SS, Chen YL, Kirsch DG, Maduekwe UN, Rosenberg AE, Nielsen GP, Sahani DV, Choy E, Harmon DC and DeLaney TF: Proton-beam, intensity-modulated, and/or intraoperative electron radiation therapy combined with aggressive anterior surgical resection for retroperitoneal sarcomas. Ann Surg Oncol 17: 1515-1529, 2010.
Received January 25, 2018

Revised February 19, 2018

Accepted February 21, 2018 